\title{
Segmental- and Normal-Mode Dielectric Relaxation of Poly(propylene glycol) under Pressure
}

\author{
C. M. ROLAND, ${ }^{1}$ T. PSUReK, ${ }^{2}$ S. PAWLUS, ${ }^{2}$ M. PALUCH ${ }^{2}$ \\ ${ }^{1}$ Naval Research Laboratory, Chemistry Division, Code 6120, Washington, DC 20375 \\ ${ }^{2}$ Institute of Physics, Silesian University, ul. Uniwersytecka 4, 40-007 Katowice, Poland
}

Received 14 April 2003; revised 17 July 2003; accepted 17 July 2003

\begin{abstract}
Dielectric measurements were obtained on poly(propylene glycol) (molecular weight: $4000 \mathrm{Da}$ ) at pressures in excess of $1.2 \mathrm{GPa}$. The segmental ( $\alpha$ process) and normal-mode ( $\alpha^{\prime}$ process) relaxations exhibited different pressure sensitivities of their relaxation strengths, as well as their relaxation times. Such results are contrary to previous reports, and (at least for the dielectric strength) can be ascribed to the capacity for intermolecular hydrogen-bond formation in this material. With equation-of-state measurements, the relative contributions of volume and thermal energy to the $\alpha$-relaxation times were quantified. Similar to other H-bonded liquids, temperature is the more dominant control variable, although the effect of volume is not negligible. (c) 2003 Wiley Periodicals, Inc. J Polym Sci Part B: Polym Phys 41: 3047-3052, 2003

Keywords: viscoelastic properties; relaxation; dielectric properties; glass transition
\end{abstract}

\section{INTRODUCTION}

Some noteworthy features of the dynamics of polymer melts include the non-Arrhenius temperature dependence of the segmental relaxation times and the non-Debye character of the relaxation function. Various experimental studies, involving spectroscopic, scattering, and relaxation methods, have been directed at understanding the molecular mechanisms underlying such behavior. The broad frequency range and high sensitivity of dielectric spectroscopy have led to its frequent use in the study of polymer dynamics. In those investigations, the focus is most often on the effects of temperature, although the dynamic properties can also be controlled by pressure. Pressure is less commonly used because of considerable experimental difficulties.

\footnotetext{
Correspondence to: C. M. Roland (E-mail: roland@nrl. navy.mil)

Journal of Polymer Science: Part B: Polymer Physics, Vol. 41, 3047-3052 (2003) ๑) 2003 Wiley Periodicals, Inc.
}

Dielectric spectra of polar polymers usually exhibit several relaxation peaks, whose position on the frequency scale generally depends on temperature, pressure, and sometimes chain length., ${ }^{1,2}$ The most prominent dielectric mode, designated as the $\alpha$ process, reflects segmental motions of the polymer chains and is closely connected to the dynamic glass transition. Below the glass-transition temperature $\left(T_{\mathrm{g}}\right)$ in the region where structural motions become frozen on the timescale of the experiment, there is usually a weak, broad peak because of the $\beta$ relaxation. This $\beta$ process is faster than the $\alpha$ relaxation and tends to merge with it as the temperature is increased above $T_{\mathrm{g}}$. For polymers having a dipole moment parallel to the chain backbone, another process can be detected dielectrically at lower frequencies. This is the normal mode (or $\alpha^{\prime}$ peak), attributed to motion of the whole macromolecule, and thus its characteristic frequency depends inversely on molecular weight. Finally, if there are any mobile ions in the polymeric material, an additional conductivity response is observed. 


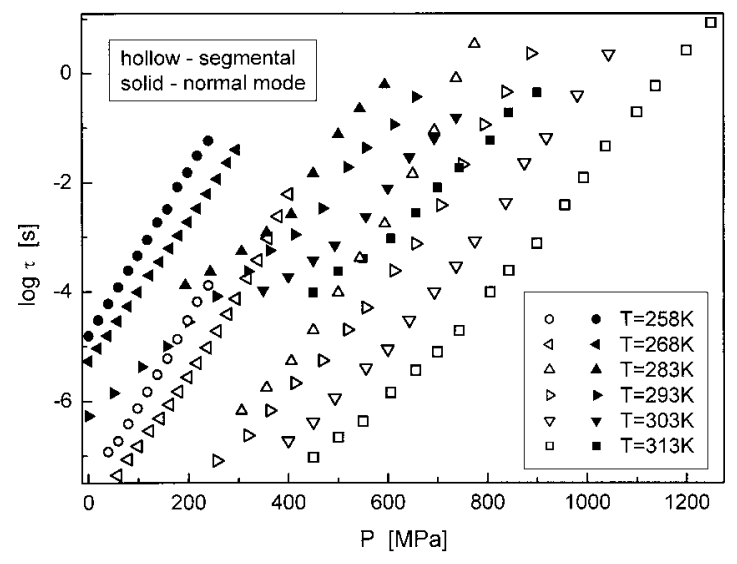

Figure 1. Relaxation times, defined from the maxima in the dielectric loss, for the segmental and normal modes at the indicated temperatures.

Dielectric relaxation in propylene glycol and polymers synthesized from it (PPG) have been the subject of many studies, ${ }^{3-12}$ including some at elevated pressure. ${ }^{13-17}$ Such interest has arisen for several reasons. The material has a large dipole moment, which makes the measurements convenient. More importantly, there is a dipole moment parallel to the chain axis, making the normal mode dielectrically active. ${ }^{18,19}$ PPG also has a higher frequency, secondary relaxation in its dielectric spectrum. ${ }^{20}$

In this article, we describe dielectric relaxation measurements of PPG under large $(>1 \mathrm{GPa})$ hydrostatic pressure. We investigate the response of both the normal and segmental relaxations to pressure. By combining the dielectric measurements with equation-of-state data for the material, we quantified the relative contribution of temperature and volume to the segmental relaxation times. This result is compared to the behavior of other hydrogen-bonded glass formers.

\section{EXPERIMENTAL}

The poly(propylene glycol) [poly(propylene oxide) with hydroxyl end groups, referred to as PPG4000] had a weight-average molecular weight of $M_{\mathrm{w}}=4000 \mathrm{Da}$, corresponding to about 70 monomer units. It was rigorously dried before all measurements and maintained in a dry nitrogen atmosphere during the dielectric experiments. The latter were carried out with a NovoControl $\mathrm{GmbH}$ dielectric spectrometer. The dielectric permittivity, $\epsilon^{*}(\omega)=\epsilon^{\prime}(\omega)-i \epsilon^{\prime \prime}(\omega)$, was measured in the frequency range from 0.1 to 3 $\times 10^{6} \mathrm{~Hz}$. For the high-pressure measurements, the parallel plate capacitor, after filling, was sealed and mounted inside a Teflon ring spacer. This assembly was then placed in the high-pressure cell. Pressure was exerted via silicone fluid, with a chamber with a piston in contact with a hydraulic press. Pressure was measured by a Nova Swiss tensometric pressure meter (resolution: $0.1 \mathrm{MPa}$ ). The temperature was controlled to $0.1 \mathrm{~K}$ with liquid flowing from a thermostatic bath.

\section{RESULTS AND DISCUSSION}

Figure 1 shows the relaxation times, $\tau_{\alpha}$ and $\tau_{\mathrm{N}}$, for the segmental and normal modes, respectively. These were taken from the frequency of the maximum in the dielectric loss. The two relaxations are increasingly separated in time with increasing molecular weight, ${ }^{15}$ and for PPG4000, the large separation makes it difficult to observe both peaks under the same conditions of $T$ and $P$. However, with increasing pressure, $\tau_{\alpha}$ and $\tau_{\mathrm{N}}$ become relatively close. This is seen most clearly in a plot of the respective activation volumes, $\Delta V=$ $\left.2.303 R T(d \log \tau / d P)\right|_{T}$ for the two processes (Fig. 2). These were calculated from the data in Figure 1 for lower pressures, for which the logarithm of the relaxation times is proportional to pressure.

The activation volume decreased with temperature, as usually found. More interesting is the

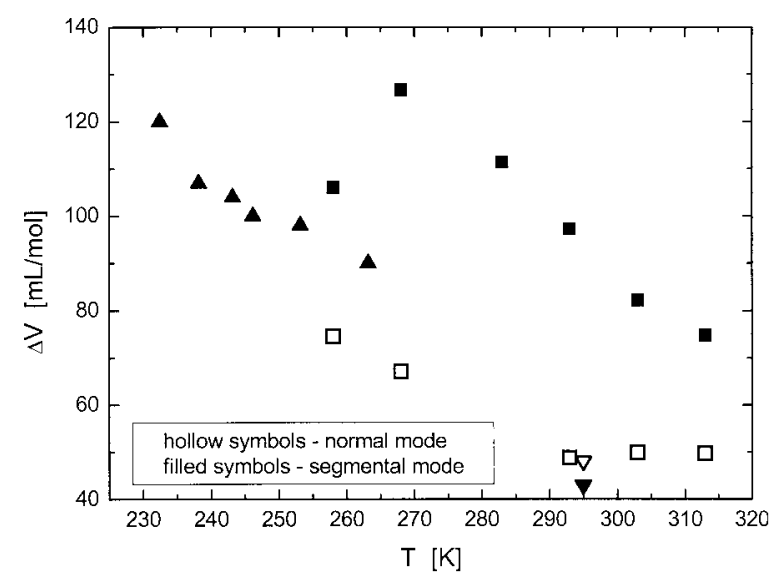

Figure 2. Activation volumes determined herein for segmental (ם) and normal ( $\square$ ) modes in PPG4000. Also shown are the values reported by Andersson and Andersson $^{15}$ for the segmental $(\boldsymbol{\nabla})$ and normal $(\nabla)$ modes and by Williams ${ }^{12}$ for the segmental mode $(\boldsymbol{\Delta})$. 


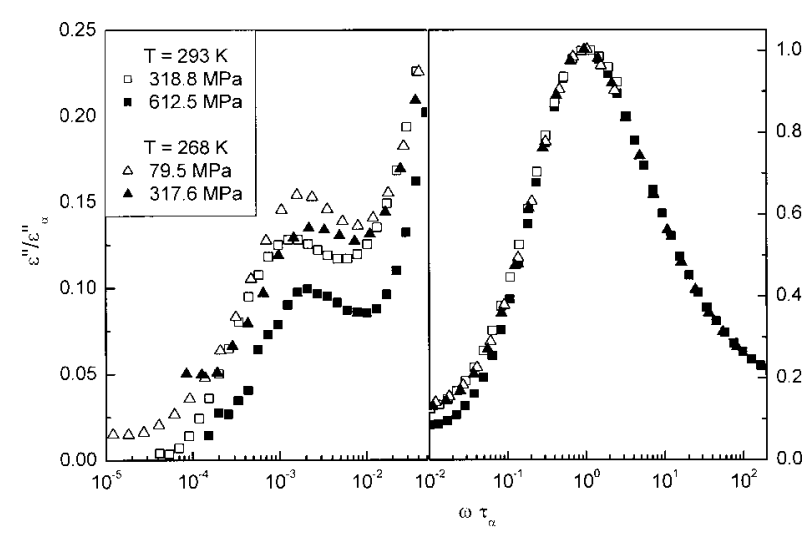

Figure 3. Normal (left panel) and segmental (right panel) modes at the indicated temperatures and pressures. The spectra were horizontally and vertically shifted to superimpose the higher-frequency segmental relaxation peaks. Note the expanded ordinate scale for the normal modes.

substantially larger values of $\Delta V$ for segmental relaxation as compared with the normal mode. This differs from Andersson and Andersson ${ }^{16}$ who reported that the normal mode is more sensitive to pressure than the segmental mode. As seen in Figure 2, our values of activation volume for the normal mode agree; the discrepancy is for $\Delta V$ for segmental relaxation. However, as seen in Figure 2 , the results of Williams ${ }^{13}$ for the latter are close to our own.

The larger activation volume for the segmental process enables resolution of the normal mode at high pressure. This is evident in Figure 3 in representative spectra in which segmental peaks measured at different conditions have been superposed by small vertical and horizontal shifts. Thus, over a broad range of $T$ and $P$, the shape of the segmental relaxation function is constant for a fixed value of the relaxation time. This is consistent with a more general conformity to timetemperature-pressure superpositioning, as reported by Williams ${ }^{13}$ for pressures up to $280 \mathrm{MPa}$. However, Suzuki et al. ${ }^{15}$ found that the $\alpha$ peak for PPG $\left(M_{\mathrm{w}}=3000 \mathrm{Da}\right)$ broadens somewhat as temperature is reduced or pressure is increased. The latter was observed for $P \leq 600 \mathrm{MPa}$.

Toward lower frequencies in Figure 3 resides the loss peak for the normal mode. The partial encroachment by the $\alpha$ peak occurs as a result of the stronger pressure dependence of the latter. Similarly, for 1,4-polyisoprene, $\tau_{\alpha}$ was more sensitive to pressure than $\tau_{\mathrm{N}}{ }^{21}$ Also, in Figure 3 scaling the heights to superimpose the segmental relaxation peaks does not superpose the peaks for the normal mode, that is, the respective strengths of the two processes also exhibit a different response to pressure.

This is illustrated in Figure 4 where the dielectric strength, $\Delta \epsilon$, for the two processes is plotted as a function of pressure for two temperatures, 258 and $268 \mathrm{~K}$. The segmental mode shows the expected response, an increase in dielectric strength with pressure, $d \Delta \epsilon_{\alpha} / d P \sim 0.002 \mathrm{MPa}^{-1}$. To some extent, this reflects a higher density; however, the increase in $\Delta \epsilon_{\alpha}$ in Figure 4 is about threefold higher than can be accounted for merely because of the densification of the material with pressure. This is similar to the anomaly found for the temperature dependence of the dielectric strength of polymers and molecular glass formers; $d \Delta \epsilon_{\alpha} / d T$ is much larger than can be accounted for from changes in density. ${ }^{22}$ This is usually taken to indicate the role of correlation effects. For materials capable of hydrogen bonding, orientational correlations among the individual dipoles can either enhance or reduce the net dipole moment. Because pressure reduces $\mathrm{H}$ bonding, ${ }^{23,24}$ the inference from Figure 4 is that the specific interactions in PPG4000 reduce the observed dielectric strength.

As seen in Figure 4, the pressure coefficient of the dielectric strength for the normal mode is two orders of magnitude smaller than for the segmental mode, and has a negligible pressure dependence. The latter implies the coil dimension is unaffected by pressure. For 1,4-polyisoprene, a strictly van der Waals polymer, the opposite result was obtained, $d \Delta \epsilon_{\alpha} / d P<d \Delta \epsilon_{\mathrm{N}} /$

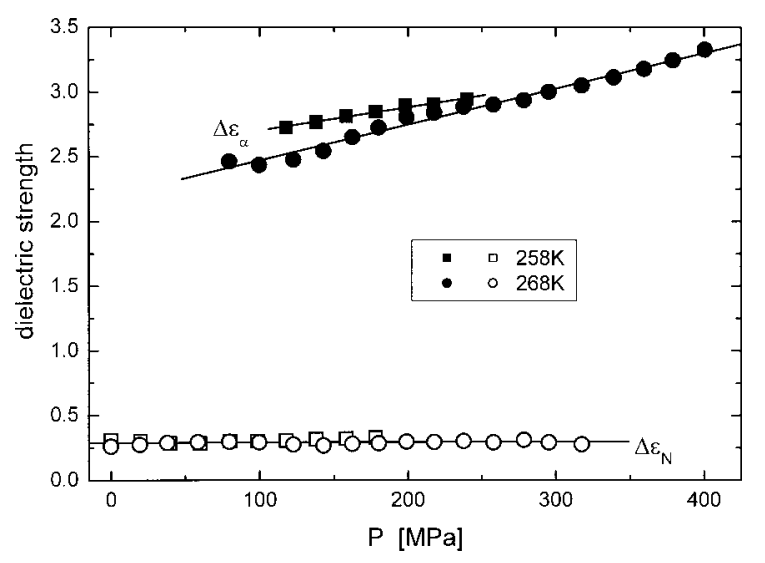

Figure 4. Pressure dependence of the dielectric strength for segmental (solid symbols) and normal (hollow) modes at the indicated temperatures. 


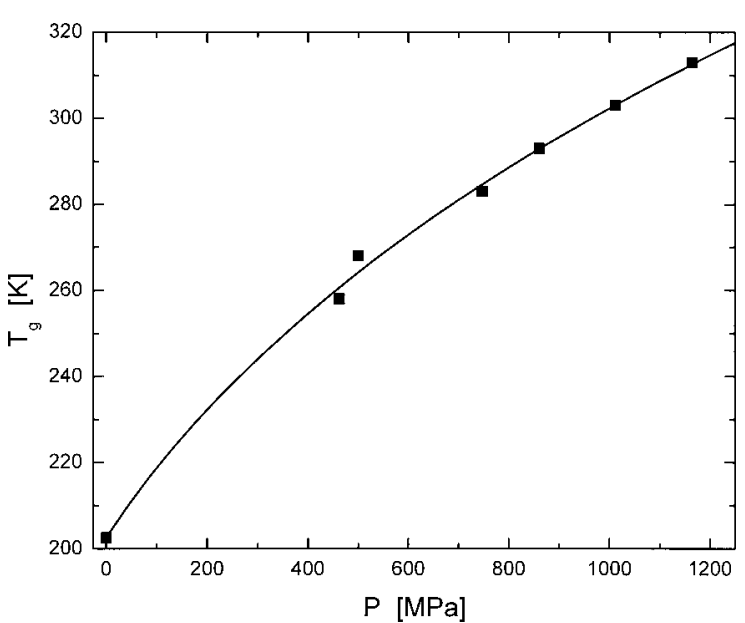

Figure 5. Temperature at which local segmental relaxation time equals $1 \mathrm{~s}$ versus pressure.

$d P,{ }^{21}$ suggesting the behavior herein is a consequence of the intermolecular association $(\mathrm{H}$ bonding) specific to the poly(propylene glycol) chain ends.

Defining the glass transition as the temperature at which the dielectric relaxation time equals $1 \mathrm{~s}$ (which avoids extrapolation of the measured data), we obtained the $T_{\mathrm{g}}$ 's displayed in Figure 5. Fitting the data to the empirical equation ${ }^{16,25}$

$$
T_{\mathrm{g}}=k_{1}\left(1+\frac{k_{2}}{k_{3}} P\right)^{1 / k_{2}}
$$

we obtain $k_{1}=202.5 \pm 2.4 \mathrm{~K}, k_{2}=3.51 \pm 0.43$, and $k_{3}=1140 \pm 140 \mathrm{MPa}^{-1}$. In the limit of zero pressure, $d T_{\mathrm{g}} / d P=0.177 \pm 0.036 \mathrm{~K} / \mathrm{MPa}$. The error is large because of the gap between the ambient pressure datum $\left(T_{\mathrm{g}}=202.5 \mathrm{~K}\right)$ and the measurements at elevated pressure $\left(T_{\mathrm{g}} \geq 258 \mathrm{~K}\right)$. Our value for the pressure coefficient of $T_{\mathrm{g}}$ is intermediate between the result of Williams ${ }^{13}$ from dielectric measurements on a poly(propylene glycol) of 300 -fold higher molecular weight, $d T_{\mathrm{g}} / d P=0.138 \mathrm{~K} / \mathrm{MPa}$, and of Andersson and Andersson, ${ }^{16}$ who obtained $d T_{\mathrm{g}} / d P=0.192$ $\mathrm{K} / \mathrm{MPa}$ from thermal-conductivity measurements on the same material as herein. Dielectric measurements on a copolymer of poly(propylene oxide) and allyl glycidyl ether yielded the same value as herein, $0.170 \mathrm{MPa} / \mathrm{K}^{14}$

Pressure-volume-temperature (PVT) data for PPG4000 were reported by Zoller and Walsh. ${ }^{26}$ We parameterized these measurements with the Tait equation ${ }^{27}$

$$
\begin{aligned}
V(T, P)= & \left(a_{0}+a_{1} T+a_{2} T^{2}\right) \\
& \times\left[1-0.0894 \ln \left(1+\frac{P}{b_{0} \exp -b_{1} T}\right)\right]
\end{aligned}
$$

The results were $a_{0}=0.9852 \mathrm{~mL} \mathrm{~g}^{-1}, a_{1}=7.2$ $\times 10^{-4} \mathrm{~mL} \mathrm{~g}^{-1} \mathrm{C}^{-1}, a_{2}=4.7 \times 10^{-7} \mathrm{~mL} \mathrm{~g}^{-1} \mathrm{C}^{-2}$, $b_{0}=171 \mathrm{MPa}$, and $b_{1}=5.2 \times 10^{-3} \mathrm{C}^{-1}$.

We can use this information to calculate the thermal-expansion coefficient, both for constant pressure, $\alpha_{P}=-V^{-1}(\partial V / \partial T)_{P}$, and for the constant value of the $\alpha$-relaxation time, $\alpha_{\tau}=-V^{-1}$ $(\partial V / \partial T)_{\tau^{*}}$. The ratio $\left|\alpha_{\tau}\right| / \alpha_{P}$ is much larger than unity if temperature is the variable governing the temperature dependence of the relaxation times, but less than one if volume exerts a stronger influence than thermal energy. ${ }^{28}$ In Figure 6, we plotted the density as a function of temperature for both constant pressure and fixed $\tau_{\alpha}$. At least near $T_{\mathrm{g}}$, the data are linear, yielding $\alpha_{\tau}=-1.40$ $\times 10^{-3}$ and $\alpha_{P}=6.97 \times 10^{-4}$, with a ratio equal to $2.0 \pm 0.4$.

Another measure of the relative contribution of thermal energy and volume is the ratio of the activation energy at constant volume, $E_{V}$ $=\left.R\left(\partial \ln \tau / \partial T^{-1}\right)\right|_{V}$, to that at constant pressure, $E_{p}=\left.R\left(\partial \ln \tau / \partial T^{-1}\right)\right|_{\mathrm{p} .}{ }^{29}$ A large ratio of $E_{V} / E_{P}$ connotes a more significant influence of temperature rather than volume of $\tau_{\alpha}$. We calculate this quantity with the relation ${ }^{30}$

$$
E_{V} / E_{p}=1-\left.\gamma \frac{\partial T}{\partial P}\right|_{\tau}
$$

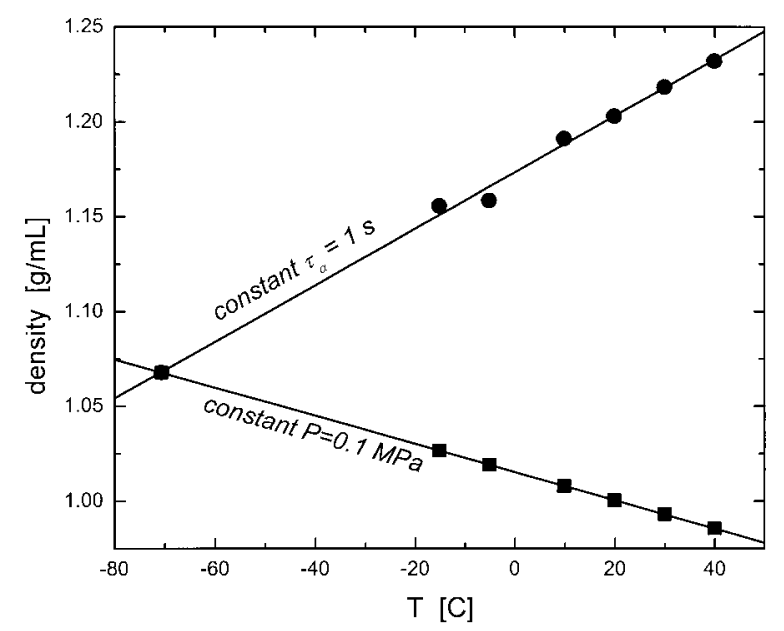

Figure 6. Thermal-expansion coefficients for isochronic $(\mathbf{O})$ and isobaric $(\mathbf{\Delta})$ conditions. 
Table 1. Comparison of Hydrogen-Bonded Glass Formers

\begin{tabular}{lcccc}
\hline & $T_{\mathrm{g}}$ & $\left|\alpha_{\tau}\right| \alpha_{\mathrm{P}}$ & $E_{V} / E_{P}$ & Ref. \\
\hline Salol & 220 & 0.9 & 0.43 & 32 \\
PPG4000 & 203 & 2.0 & 0.66 & Herein \\
Sorbitol & 273 & 6 & 0.87 & 22 \\
Glycerol & 189 & 17 & - & 27 \\
\hline
\end{tabular}

where $\gamma$ is the thermal-pressure coefficient and, as described above, $\left.(\partial T / \partial P)\right|_{\tau}=d T_{\mathrm{g}} / d P$. From the PVT data, we calculate $\gamma=1.915 \pm 0.009 \mathrm{MPa} / \mathrm{K}$. With $d T_{\mathrm{g}} / d P=0.177 \mathrm{~K} / \mathrm{MPa}$, the result is $E_{V} / E_{P}$ $=0.66 \pm 0.07$. This agrees within the error with the value of the ratio obtained by Williams, ${ }^{13}$ from dielectric measurements on a poly(propylene glycol) of 300-fold higher molecular weight The magnitude of $E_{V} / E_{P}$ is entirely consistent with the ratio of the thermal-expansion coefficients. Both indicate that volume and temperature influence the relaxation times, with the latter having a larger contribution.

We have recently shown that for van der Waals liquids and polymers, local $\alpha$-relaxation times are governed almost equally by volume and temperature. ${ }^{31,32}$ The exceptions to this seem to be hydrogen-bonded materials, which include propylene glycol. In Table 1 we have collected results for four H-bonded glass formers. ${ }^{23,28,33}$ The expansivity and activation energy ratios were taken from the original publications or calculated from the data therein. All values are for the equilibrium liquid near $T_{\mathrm{g}}\left[1 \leq \tau_{\alpha}(\mathrm{s}) \leq 100\right]$; these ratios change less than $10 \%$ for an order of magnitude change in $\tau_{\alpha}$. In the table the relative degree to which $\tau_{\alpha}(T)$ is controlled by temperature, rather than density, parallels the extent of intermolecular hydrogen bonding. Thus, the $\mathrm{H}$ bonding in salol is primarily intramolecular, and $\left|\alpha_{\tau}\right| / \alpha_{p}$ is less than one, signifying that volume is more important than thermal energy. For PPG4000, only the terminal chain units, which are less than $3 \%$ of the total, can form hydrogen bonds; accordingly, $\left|\alpha_{\tau}\right| / \alpha_{p}$ is not very large, and volume contributes to the temperature dependence of $\tau_{\alpha}$. However, for sorbitol and glycerol, the expansivity ratio becomes very large in proportion to the degree of $\mathrm{H}$ bonding. The extensive $\mathrm{H}$ bonding of the polyalcohols causes temperature to become the dominant control variable.

The reason for this behavior is the competing effects of volume and temperature changes on associated liquids. ${ }^{32}$ Compression reduces $\mathrm{H}$ bonding, ${ }^{34,35}$ which partially counters the increase of $\tau_{\alpha}$ because of the reduced volume. Consequently, the net effect of volume is weak. Temperature changes have the opposite effect. Lower thermal energy enhances $\mathrm{H}$ bonding, which contributes further to the slowing down of molecular motion as temperature is reduced. Thus, temperature dominates the response of glass formers whose structural relaxation is affected significantly by the presence of hydrogen bonding.

\section{CONCLUSIONS}

Our main findings herein are as follows.

First, the pressure sensitivity of segmental relaxation ( $\alpha$ process) in PPG4000 was significantly greater than for the normal modes, contrary to some previous research. The differing response to pressure enables resolution of the respective peaks in the dielectric loss for the segmental and normal modes.

Second, the dielectric strength for segmental relaxation displayed the usual behavior, an increase with increasing pressure, $d \Delta \epsilon_{\alpha} / d P \sim 0.002$ $\mathrm{MPa}^{-1}$. However, the strength of the normal mode was essentially invariant to pressure. Thus, the relative strength of the two modes varied oppositely with pressure than reported previously for nonassociated polymers.

Third, the temperature dependence of the $\alpha$-relaxation times were governed both by volume and temperature, although the latter exerted a somewhat stronger influence. The magnitude of $\left|\alpha_{\tau}\right| / \alpha_{\mathrm{P}}$ for PPG4000 was consistent with its limited degree of hydrogen bonding, when comparing the ratio of the isochoric and isobaric thermal-expansion coefficients.

The authors thank V. Mayrantzas for helpful comments. The work at NRL was supported by the Office of Naval Research and that at Silesian University by the State Committee for Scientific Research (KBN, Poland) under Project 2PO3B 03323.

\section{REFERENCES AND NOTES}

1. Mc Crum, N. G.; Read, B. E.; Williams, G. Anelastic and Dielectric Effects in Polymeric Solids; Dover: New York, 1967.

2. Broadband Dielectric Spectroscopy; Kremer, F.; Schönhals, A., Eds.; Springer-Verlag: Berlin, 2003. 
3. Yano, S.; Rahalkar, R. R.; Hunter, S. P.; Wang, C. H.; Boyd, R. H. J Polym Sci Polym Phys Ed 1976 , 14, 1877.

4. Beevers, M. S.; Elliott, D. A.; Williams, G. Polymer 1980, 21, 13.

5. Beevers, M. S.; Elliott, D. A.; Williams, G. Polymer 1979, 20, 785.

6. Varadarajan, K.; Boyer, R. F. Polymer 1982, 23, 314.

7. Johari, G. P.; Pathmanathan, K. J Chem Phys 1986, 85, 6811.

8. Stickel, F. Ph.D. Thesis, Mainz University, Germany; Shaker: Aachen, 1995.

9. Schönhals, A.; Kremer, F.; Schlosser, E. Prog Colloid Polym Sci 1993, 91, 39.

10. Schönhals, A.; Stauga, R. J Non-Cryst Solids 1998, 235-237, 450.

11. León, C.; Ngai, K. L.; Roland, C. M. J Chem Phys 1999, 110, 11585.

12. Mattsson, J.; Bergman, R.; Jacobsson, P.; Börjesson, L. Phys Rev Lett 2003, 90, 075702.

13. Williams, G. Trans Faraday Soc 1965, 61, 1564.

14. Fontanella, J. J.; Wintersgill, M. C.; Smith, M. K.; Semancik, J.; Andeen, C. G. J Appl Phys 1986, 60, 2665.

15. Suzuki, A.; Masuko, M.; Wakisaka, K. Tribology Int 2002, 35, 55.

16. Andersson, S. P.; Andersson, O. Macromolecules 1998, 31, 2999.

17. Casalini, R.; Roland, C. M. Phys Rev Lett 2003, 91, 15702.

18. Baur, M. E.; Stockmayer, W. H. J Chem Phys 1965, 43, 4319 .

19. Alper, T.; Barlow, A. J.; Gray, R. W. Polymer 1976, $17,665$.

20. Johari, G. P. Polymer 1986, 26, 866.
21. Floudas, G.; Reisinger, T. J Chem Phys 1999, 111, 5201.

22. Schönhals, A. Broadband Dielectric Spectroscopy; Kremer, F.; Schönhals, A., Eds.; Springer-Verlag: Berlin, 2003; Chapter 7.

23. Hensel-Bielowka, S.; Paluch, M.; Roland, C. M. J Phys Chem B 2002, 106, 12459.

24. Roland, C. M.; Casalini, R.; Paluch, M. Chem Phys Lett 2003, 367, 259.

25. Roland, C. M.; Casalini, R. Macromolecules 2003, 36, 1361.

26. Zoller, P.; Walsh, D. J. Standard Pressure-VolumeTemperature Data for Polymers; Technomic: Lancaster, PA, 1995, pp 241-242.

27. Orwoll, R. A. In Physical Properties of Polymers Handbook; Mark, J. E., Ed.; American Institute of Physics: Woodbury, NY, 1996; Chapter 7.

28. Ferrer, M. L.; Lawrence, Ch.; Demirjian, B. G.; Kivelson, D.; Alba-Simonesco, Ch.; Tarjus, G. J Chem Phys 1998, 109, 8010.

29. Williams, G. In Dielectric Spectroscopy of Polymeric Materials; Runt, J. P.; Fitzgerald, J. J., Eds.; American Chemical Society: Washington, DC, 1997, pp 3-53.

30. Naoki, M.; Endou, H.; Matsumoto, K. J Phys Chem 1987, 91, 4169.

31. Paluch, M.; Casalini, R.; Roland, C. M. Phys Rev B 2002, 66, 092202.

32. Roland, C. M.; Paluch, M.; Pakula, T.; Casalini, R. Philosophical Magazine B, in press.

33. Casalini, R.; Roland, C. M.; Paluch, M. J Phys Chem A 2003, 107, 2369.

34. Naoki, M.; Katahira, S. J Phys Chem 1991, 95, 431.

35. Poole, P. H.; Sciortino, F.; Grande, T.; Stanley, H. E.; Angell, C. A. Phys Rev Lett 1994, 73, 1632. 